\title{
Supplementary Information for FaIRv2.0.0: a generalised impulse-response model for climate uncertainty and future scenario exploration
}

Nicholas J. Leach ${ }^{1}$, Stuart Jenkins ${ }^{1}$, Zebedee Nicholls ${ }^{2,3}$, Christopher J. Smith ${ }^{4,5}$, John Lynch ${ }^{1}$, Michelle Cain $^{1}$, Tristram Walsh ${ }^{1}$, Bill Wu ${ }^{1}$, Junichi Tsutsui ${ }^{6}$, and Myles R. Allen ${ }^{1,7}$

${ }^{1}$ Department of Physics, Atmospheric, Oceanic, and Planetary Physics, University of Oxford, United Kingdom.

${ }^{2}$ Australian-German Climate and Energy College, University of Melbourne, Australia.

${ }^{3}$ School of Earth Sciences, University of Melbourne, Australia.

${ }^{4}$ School of Earth and Environment, University of Leeds, Leeds, UK.

${ }^{5}$ International Institute for Applied Systems Analysis, Laxenburg, Austria.

${ }^{6}$ Environmental Science Laboratory, Central Research Institute of Electric Power Industry, Abiko-shi, Japan.

${ }^{7}$ Environmental Change Institute, University of Oxford, Oxford, UK.

Correspondence: Nicholas J. Leach (nicholas.leach@stx.ox.ac.uk)

Copyright statement. @ Author(s) 2019. This work is distributed under the Creative Commons Attribution 4.0 License. 
Table S1. Units used in GIR when the default parameter set is used for each gas or aerosol species. Default forcing unit for all species is $\mathrm{Wm}^{-2}$

\begin{tabular}{lrrrrrrrrrr}
\hline Variable & $\mathrm{CO}_{2}$ & $\mathrm{CH}_{4}$ & $\mathrm{~N}_{2} \mathrm{O}$ & $\mathrm{SOx}$ & $\mathrm{NOx}$ & $\mathrm{BC}$ & $\mathrm{OC}$ & $\mathrm{NH} 3$ & $\mathrm{VOC}$ & All other WMGHGs \\
\hline Emissions & $\mathrm{PgC}$ & $\mathrm{TgCH}_{4}$ & $\mathrm{TgN}$ & $\mathrm{TgSO}_{2}$ & $\mathrm{TgN}$ & $\mathrm{TgC}$ & $\mathrm{TgC}$ & $\mathrm{Tg}$ & $\mathrm{Tg}$ & $\mathrm{Tg}$ \\
Concentrations & $\mathrm{ppm}$ & $\mathrm{ppb}$ & $\mathrm{ppb}$ & - & - & - & - & - & - & $\mathrm{ppb}$ \\
\hline
\end{tabular}




\section{FaIRv2.0 parameter defaults}

Table S2. FaIRv2.0 default parameter values

\begin{tabular}{|c|c|c|c|c|c|c|c|c|c|c|c|c|c|c|c|c|c|}
\hline & $a_{1}$ & $a_{2}$ & $a_{3}$ & $a_{4}$ & $\tau_{1}$ & $\tau_{2}$ & $\tau_{3}$ & $\tau_{4}$ & $r_{0}$ & $r_{u}$ & $T$ & $r_{a}$ & PI_conc & emis2conc & $f_{1}$ & $f_{2}$ & $f_{3}$ \\
\hline bc & 1.0 & 0 & 0 & 0 & 1.0 & 1.0 & 1.0 & 1.0 & 1.0 & 0 & 0 & 0 & - & 1.0 & 0 & 0.03222 & 0 \\
\hline bclaci & - & - & - & - & - & - & - & - & - & - & - & - & - & - & 0 & -0.0104 & 0 \\
\hline bclbc_on_snow & - & - & - & - & - & - & - & - & - & - & - & - & - & - & 0 & 0.0113 & 0 \\
\hline $\mathrm{c} 2 \mathrm{f} 6$ & 1.0 & 0 & 0 & 0 & 100.0 & 1.0 & 1.0 & 1.0 & 184.9 & 0 & 0 & 0 & - & 0.04077 & 0 & 0.25 & 0 \\
\hline $\mathrm{c} 3 \mathrm{f} 8$ & 1.0 & 0 & 0 & 0 & 26.0 & 1.0 & 1.0 & 1.0 & 132.8 & 0 & 0 & 0 & - & 0.02993 & 0 & 0.28 & 0 \\
\hline c4f10 & 1.0 & 0 & 0 & 0 & 26.0 & 1.0 & 1.0 & 1.0 & 132.8 & 0 & 0 & 0 & - & 0.02364 & 0 & 0.36 & 0 \\
\hline$c 5 f 12$ & 1.0 & 0 & 0 & 0 & 41.0 & 1.0 & 1.0 & 1.0 & 169.6 & 0 & 0 & 0 & - & 0.01953 & 0 & 0.41 & 0 \\
\hline c6f14 & 1.0 & 0 & 0 & 0 & 31.0 & 1.0 & 1.0 & 1.0 & 148.6 & 0 & 0 & 0 & - & 0.01664 & 0 & 0.44 & 0 \\
\hline c7f 16 & 1.0 & 0 & 0 & 0 & 30.0 & 1.0 & 1.0 & 1.0 & 145.7 & 0 & 0 & 0 & - & 0.0145 & 0 & 0.5 & 0 \\
\hline c8f18 & 1.0 & 0 & 0 & 0 & 30.0 & 1.0 & 1.0 & 1.0 & 145.7 & 0 & 0 & 0 & - & 0.01284 & 0 & 0.55 & 0 \\
\hline c_c4f8 & 1.0 & 0 & 0 & 0 & 32.0 & 1.0 & 1.0 & 1.0 & 151.3 & 0 & 0 & 0 & - & 0.02813 & 0 & 0.32 & 0 \\
\hline carbon_dioxide & 0.2173 & 0.224 & 0.2824 & 0.2763 & inf & 394.4 & 36.54 & 4.304 & 30.4 & 0.0177 & 2.64 & 0 & 278.0 & 0.4689 & 4.57 & 0 & 0.086 \\
\hline carbon_tetrachloride & 1.0 & 0 & 0 & 0 & 0.32 & 1.0 & 1.0 & 1.0 & 1.794 & 0 & 0 & 0 & $2.5 \mathrm{e}-05$ & 0.03658 & 0 & 0.174 & 0 \\
\hline carbon_tetrachloridelstrat_03 & - & - & - & - & - & - & - & - & - & - & - & - & - & - & 0 & -0.07699 & 0 \\
\hline cf4 & 1.0 & 0 & 0 & 0 & 500.0 & 1.0 & 1.0 & 1.0 & 131.0 & 0 & 0 & 0 & 0.03405 & 0.06394 & 0 & 0.09 & 0 \\
\hline cfc11 & 1.0 & 0 & 0 & 0 & 0.52 & 1.0 & 1.0 & 1.0 & 2.915 & 0 & 0 & 0 & - & 0.04096 & 0 & 0.26 & 0 \\
\hline cfc113 & 1.0 & 0 & 0 & 0 & 0.93 & 1.0 & 1.0 & 1.0 & 5.213 & 0 & 0 & 0 & - & 0.03003 & 0 & 0.3 & 0 \\
\hline cfc113|strat_o3 & - & - & - & - & - & - & - & - & - & - & - & - & - & - & 0 & -0.02411 & 0 \\
\hline cfc114 & 1.0 & 0 & 0 & 0 & 1.89 & 1.0 & 1.0 & 1.0 & 10.59 & 0 & 0 & 0 & - & 0.03292 & 0 & 0.31 & 0 \\
\hline cfc114|strat_03 & - & - & - & - & - & - & - & - & - & - & - & - & - & - & 0 & -0.007323 & 0 \\
\hline $\operatorname{cfc} 115$ & 1.0 & 0 & 0 & 0 & 5.4 & 1.0 & 1.0 & 1.0 & 30.27 & 0 & 0 & 0 & - & 0.03643 & 0 & 0.2 & 0 \\
\hline cfc115/strat_o3 & - & - & - & - & - & - & - & - & - & - & - & - & - & - & 0 & -0.001333 & 0 \\
\hline cfc11lstrat_03 & - & - & - & - & - & - & - & - & - & - & - & - & - & - & 0 & -0.05324 & 0 \\
\hline cfc12 & 1.0 & 0 & 0 & 0 & 1.02 & 1.0 & 1.0 & 1.0 & 5.717 & 0 & 0 & 0 & - & 0.04654 & 0 & 0.32 & 0 \\
\hline cfc12|strat_o3 & - & - & - & - & - & - & - & - & - & - & - & - & - & - & 0 & -0.0209 & 0 \\
\hline $\operatorname{ch} 2 \mathrm{cl} 2$ & 1.0 & 0 & 0 & 0 & 0.004928 & 1.0 & 1.0 & 1.0 & 0.02762 & 0 & 0 & 0 & 0.006913 & 0.06625 & 0 & 0.028 & 0 \\
\hline $\operatorname{ch} 3 \operatorname{ccl} 3$ & 1.0 & 0 & 0 & 0 & 0.05 & 1.0 & 1.0 & 1.0 & 0.2803 & 0 & 0 & 0 & - & 0.04218 & 0 & 0.07 & 0 \\
\hline ch3ccl3|strat_o3 & - & - & - & - & - & - & - & - & - & - & - & - & - & - & 0 & -0.08305 & 0 \\
\hline chcl3 & 1.0 & 0 & 0 & 0 & 0.00501 & 1.0 & 1.0 & 1.0 & 0.02808 & 0 & 0 & 0 & 0.006 & 0.04714 & 0 & 0.07 & 0 \\
\hline co & 1.0 & 0 & 0 & 0 & 1.0 & 1.0 & 1.0 & 1.0 & 1.0 & 0 & 0 & 0 & - & 1.0 & 0 & 0 & 0 \\
\hline coltrop_03 & - & - & - & - & - & - & - & - & - & - & - & - & - & - & 0 & 0.00012 & 0 \\
\hline halon1202 & 1.0 & 0 & 0 & 0 & 0.025 & 1.0 & 1.0 & 1.0 & 0.1401 & 0 & 0 & 0 & - & 0.02682 & 0 & 0.27 & 0 \\
\hline halon1202|strat_03 & - & - & - & - & - & - & - & - & - & - & - & - & - & - & 0 & -1.882 & 0 \\
\hline halon1211 & 1.0 & 0 & 0 & 0 & 0.16 & 1.0 & 1.0 & 1.0 & 0.8968 & 0 & 0 & 0 & $4.447 \mathrm{e}-06$ & 0.03403 & 0 & 0.29 & 0 \\
\hline halon1211/strat_03 & - & - & - & - & - & - & - & - & - & - & - & - & - & - & 0 & -1.263 & 0 \\
\hline halon1301 & 1.0 & 0 & 0 & 0 & 0.72 & 1.0 & 1.0 & 1.0 & 4.036 & 0 & 0 & 0 & - & 0.03779 & 0 & 0.3 & 0 \\
\hline halon1301|strat_o3 & - & - & - & - & - & - & - & - & - & - & - & - & - & - & 0 & -0.6575 & 0 \\
\hline halon2402 & 1.0 & 0 & 0 & 0 & 0.28 & 1.0 & 1.0 & 1.0 & 1.569 & 0 & 0 & 0 & - & 0.02166 & 0 & 0.31 & 0 \\
\hline halon24021strat_o3 & - & - & - & - & - & - & - & - & - & - & - & - & - & - & 0 & -1.552 & 0 \\
\hline hcfc141b & 1.0 & 0 & 0 & 0 & 0.094 & 1.0 & 1.0 & 1.0 & 0.5269 & 0 & 0 & 0 & - & 0.04812 & 0 & 0.16 & 0 \\
\hline hcfc141blstrat_o3 & - & - & - & - & - & - & - & - & - & - & - & - & - & - & 0 & -0.02533 & 0 \\
\hline hcfc142b & 1.0 & 0 & 0 & 0 & 0.18 & 1.0 & 1.0 & 1.0 & 1.009 & 0 & 0 & 0 & - & 0.05599 & 0 & 0.19 & 0 \\
\hline hcfc142blstrat_o3 & - & - & - & - & - & - & - & - & - & - & - & - & - & - & 0 & -0.006152 & 0 \\
\hline $\mathrm{hcfc} 22$ & 1.0 & 0 & 0 & 0 & 0.119 & 1.0 & 1.0 & 1.0 & 0.667 & 0 & 0 & 0 & - & 0.06507 & 0 & 0.21 & 0 \\
\hline hcfc221strat_03 & - & - & - & - & - & - & - & - & - & - & - & - & - & - & 0 & -0.006746 & 0 \\
\hline hfc125 & 1.0 & 0 & 0 & 0 & 0.3 & 1.0 & 1.0 & 1.0 & 1.682 & 0 & 0 & 0 & - & 0.04688 & 0 & 0.23 & 0 \\
\hline hfc134a & 1.0 & 0 & 0 & 0 & 0.14 & 1.0 & 1.0 & 1.0 & 0.7847 & 0 & 0 & 0 & - & 0.05515 & 0 & 0.16 & 0 \\
\hline hfc143a & 1.0 & 0 & 0 & 0 & 0.51 & 1.0 & 1.0 & 1.0 & 2.859 & 0 & 0 & 0 & - & 0.06695 & 0 & 0.16 & 0 \\
\hline hfc152a & 1.0 & 0 & 0 & 0 & 0.016 & 1.0 & 1.0 & 1.0 & 0.08968 & 0 & 0 & 0 & - & 0.08519 & 0 & 0.1 & 0 \\
\hline hfc227ea & 1.0 & 0 & 0 & 0 & 0.36 & 1.0 & 1.0 & 1.0 & 2.018 & 0 & 0 & 0 & - & 0.03309 & 0 & 0.26 & 0 \\
\hline hfc23 & 1.0 & 0 & 0 & 0 & 2.28 & 1.0 & 1.0 & 1.0 & 12.78 & 0 & 0 & 0 & - & 0.08037 & 0 & 0.18 & 0 \\
\hline hfc236fa & 1.0 & 0 & 0 & 0 & 2.13 & 1.0 & 1.0 & 1.0 & 11.94 & 0 & 0 & 0 & - & 0.03701 & 0 & 0.24 & 0 \\
\hline hfc245fa & 1.0 & 0 & 0 & 0 & 0.079 & 1.0 & 1.0 & 1.0 & 0.4428 & 0 & 0 & 0 & - & 0.04197 & 0 & 0.24 & 0 \\
\hline $\mathrm{hfc} 32$ & 1.0 & 0 & 0 & 0 & 0.054 & 1.0 & 1.0 & 1.0 & 0.3027 & 0 & 0 & 0 & - & 0.1082 & 0 & 0.11 & 0 \\
\hline hfc $365 \mathrm{mfc}$ & 1.0 & 0 & 0 & 0 & 0.089 & 1.0 & 1.0 & 1.0 & 0.4989 & 0 & 0 & 0 & - & 0.038 & 0 & 0.22 & 0 \\
\hline hfc4310mee & 1.0 & 0 & 0 & 0 & 0.17 & 1.0 & 1.0 & 1.0 & 0.9529 & 0 & 0 & 0 & - & 0.02232 & 0 & 0.359 & 0 \\
\hline methane & 1.0 & 0 & 0 & 0 & 8.8 & 1.0 & 1.0 & 1.0 & 8.8 & 0 & -0.33 & 0.00032 & 720.0 & 0.3517 & 0 & 0 & 0.0385 \\
\hline methanelstrat_h2o & - & - & - & - & - & - & - & - & - & - & - & - & - & - & 0 & $5.5 \mathrm{e}-05$ & 0 \\
\hline methaneltrop_o3 & - & - & - & - & - & - & - & - & - & - & - & - & - & - & 0 & 0.000133 & 0 \\
\hline methyl_bromide & 1.0 & 0 & 0 & 0 & 0.008 & 1.0 & 1.0 & 1.0 & 0.04484 & 0 & 0 & 0 & 0.0053 & 0.05927 & 0 & 0.004 & 0 \\
\hline methyl_bromidelstrat_o3 & - & - & - & - & - & - & - & - & - & - & - & - & - & - & 0 & -1.972 & 0 \\
\hline methyl_chloride & 1.0 & 0 & 0 & 0 & 0.009 & 1.0 & 1.0 & 1.0 & 0.05045 & 0 & 0 & 0 & 0.457 & 0.1114 & 0 & 0.004 & 0 \\
\hline methyl_chloridelstrat_03 & - & - & - & - & - & - & - & - & - & - & - & - & - & - & 0 & -0.04614 & 0 \\
\hline nf3 & 1.0 & 0 & 0 & 0 & 5.69 & 1.0 & 1.0 & 1.0 & 31.89 & 0 & 0 & 0 & - & 0.07925 & 0 & 0.2 & 0 \\
\hline nh3 & 1.0 & 0 & 0 & 0 & 1.0 & 1.0 & 1.0 & 1.0 & 1.0 & 0 & 0 & 0 & - & 1.0 & 0 & 0 & 0 \\
\hline nitrous_oxide & 1.0 & 0 & 0 & 0 & 109.0 & 1.0 & 1.0 & 1.0 & 65.45 & 0 & 0 & 0 & 270.0 & 0.201 & 0 & 0 & 0.107 \\
\hline nmvoc & 1.0 & 0 & 0 & 0 & 1.0 & 1.0 & 1.0 & 1.0 & 1.0 & 0 & 0 & 0 & - & 1.0 & 0 & 0 & 0 \\
\hline nmvocltrop_o3 & - & - & - & - & - & - & - & - & - & - & - & - & - & - & 0 & 0.00026 & 0 \\
\hline nox & 1.0 & 0 & 0 & 0 & 1.0 & 1.0 & 1.0 & 1.0 & 1.0 & 0 & 0 & 0 & - & 1.0 & 0 & 0 & 0 \\
\hline nox_avi & 1.0 & 0 & 0 & 0 & 1.0 & 1.0 & 1.0 & 1.0 & 1.0 & 0 & 0 & 0 & - & 1.0 & 0 & 0 & 0 \\
\hline nox_avilcontrails & - & - & - & - & - & - & - & - & - & - & - & - & - & - & 0 & 0.0115 & 0 \\
\hline nox|trop_o3 & - & - & - & - & - & - & - & - & - & - & - & - & - & - & 0 & 0.00098 & 0 \\
\hline oc & 1.0 & 0 & 0 & 0 & 1.0 & 1.0 & 1.0 & 1.0 & 1.0 & 0 & 0 & 0 & - & 1.0 & 0 & -0.009421 & 0 \\
\hline oclaci & - & - & - & - & - & - & - & - & - & - & - & - & - & - & 0 & -0.0104 & 0 \\
\hline sf6 & 1.0 & 0 & 0 & 0 & 32.0 & 1.0 & 1.0 & 1.0 & 151.3 & 0 & 0 & 0 & - & 0.03852 & 0 & 0.57 & 0 \\
\hline so2 2 & 1.0 & 0 & 0 & 0 & 1.0 & 1.0 & 1.0 & 1.0 & 1.0 & 0 & 0 & 0 & 204.0 & 1.0 & 0 & -0.002444 & 0 \\
\hline so $2 \mathrm{f} 2$ & 1.0 & 0 & 0 & 0 & 0.36 & 1.0 & 1.0 & 1.0 & 2.018 & 0 & 0 & 0 & - & 0.05513 & 0 & 0.2 & 0 \\
\hline so2laci & - & - & - & - & - & - & - & - & - & - & - & - & - & - & -1.69 & 0 & 0 \\
\hline
\end{tabular}


Table S3. GWP metric for default parameter values computed against a baseline emission scenario that reproduces historical concentrations (Meinshausen et al., 2017) up to 2014, then the fixed 2014 value following. These are calculated using the change in ERF arising from a $1 \mathrm{t}$ emission pulse in 2015. To compute the "direct" GWP value, we use the change in the direct ERF of each agent (ie. corresponding to the parameters without a "l" in their name in table S2). To compute the "indirect" GWP value, we use the change in the total ERF arising 10 from the emission pulse of the agent. Hence the "indirect" GWP value will include effects such as changes to $\mathrm{CO}_{2}$ uptake due to differences in the temperature responses of the baseline and perturbed runs. For ozone depleting substances, the "indirect" value includes ERF changes arising from ozone depletion. For aerosols, the "direct" value includes the radiation interaction component only, while the "indirect" value also includes cloud interaction. We emphasize that these values do not represent new estimates for the value of the GWP metric and are intended for reference and model comparison. 


\begin{tabular}{|c|c|c|c|c|c|c|c|c|c|c|c|c|}
\hline $\begin{array}{l}\text { Time horizon / yrs } \\
\text { metric type } \\
\text { Gas name }\end{array}$ & $\begin{array}{l}5 \\
\text { direct }\end{array}$ & indirect & $\begin{array}{l}10 \\
\text { direct }\end{array}$ & indirect & $\begin{array}{l}20 \\
\text { direct }\end{array}$ & indirect & $\begin{array}{l}50 \\
\text { direct }\end{array}$ & indirect & $\begin{array}{l}100 \\
\text { direct }\end{array}$ & indirect & $\begin{array}{l}500 \\
\text { direct }\end{array}$ & indirect \\
\hline $\mathrm{bc}$ & 4510 & 4650 & 2610 & 2710 & 1490 & 1550 & 696 & 746 & 381 & 425 & 86.8 & 121 \\
\hline c2f6 & 7890 & 7900 & 8680 & 8700 & 9640 & 9610 & 11100 & 11100 & 12100 & 12100 & 13400 & 13600 \\
\hline $\mathrm{c} 3 \mathrm{f} 8$ & 6480 & 6490 & 7120 & 7140 & 7900 & 7870 & 9050 & 9030 & 9780 & 9790 & 10300 & 10400 \\
\hline $\mathrm{c} 4 \mathrm{f} 10$ & 6580 & 6590 & 7240 & 7250 & 8020 & 7990 & 9190 & 9170 & 9930 & 9950 & 10400 & 10500 \\
\hline c5f 12 & 6200 & 6210 & 6810 & 6830 & 7560 & 7530 & 8680 & 8660 & 9410 & 9420 & 10200 & 10300 \\
\hline c6f14 & 5660 & 5670 & 6230 & 6240 & 6910 & 6870 & 7930 & 7890 & 8570 & 8560 & 9120 & 9210 \\
\hline $\mathrm{c} 7 \mathrm{f} 16$ & 5610 & 5620 & 6170 & 6180 & 6840 & 6800 & 7840 & 7810 & 8480 & 8470 & 9000 & 9110 \\
\hline c8f 18 & 5460 & 5470 & 6010 & 6030 & 6660 & 6700 & 7640 & 7740 & 8260 & 8410 & 8770 & 9060 \\
\hline c_c4f8 & 6960 & 6970 & 7660 & 7670 & 8490 & 8460 & 9740 & 9730 & 10500 & 10600 & 11200 & 11400 \\
\hline carbon_dioxide & 1.0 & 0.999 & 1.0 & 0.997 & 1.0 & 0.994 & 1.0 & 0.989 & 1.0 & 0.987 & 1.0 & 0.981 \\
\hline carbon_tetrachloride & 4520 & 2530 & 4620 & 2580 & 4450 & 2500 & 3490 & 1980 & 2310 & 1340 & 549 & 374 \\
\hline cf4 & 4450 & 4460 & 4900 & 4920 & 5440 & 5480 & 6270 & 6350 & 6840 & 6950 & 7720 & 7950 \\
\hline $\operatorname{cfc} 11$ & 7820 & 6230 & 8210 & 6550 & 8330 & 6590 & 7430 & 5870 & 5610 & 4420 & 1490 & 1130 \\
\hline $\operatorname{cfc} 113$ & 6770 & 6240 & 7260 & 6690 & 7650 & 7000 & 7580 & 6940 & 6550 & 6000 & 2250 & 2030 \\
\hline $\operatorname{cfc} 114$ & 7790 & 7610 & 8460 & 8280 & 9150 & 8910 & 9770 & 9530 & 9420 & 9230 & 4830 & 4820 \\
\hline $\operatorname{cfc} 115$ & 5610 & 5580 & 6150 & 6120 & 6760 & 6680 & 7590 & 7500 & 7910 & 7840 & 6400 & 6430 \\
\hline $\operatorname{cfc} 12$ & 11200 & 10500 & 12100 & 11200 & 12800 & 11800 & 12800 & 11800 & 11300 & 10400 & 4060 & 3720 \\
\hline $\operatorname{ch} 2 \mathrm{cl} 2$ & 129 & 129 & 74.2 & 74.7 & 42.2 & 42.9 & 19.8 & 20.6 & 10.8 & 11.7 & 2.46 & 3.34 \\
\hline $\operatorname{ch} 3 \mathrm{ccl} 3$ & 1380 & -258.0 & 1050 & -197.0 & 670 & -126.0 & 319 & -61.5 & 175 & -34.9 & 39.8 & -9.75 \\
\hline $\operatorname{chcl} 3$ & 233 & 233 & 134 & 135 & 76.3 & 77.6 & 35.7 & 37.3 & 19.6 & 21.2 & 4.45 & 6.04 \\
\hline co & 0 & 16.9 & 0 & 9.8 & 0 & 5.63 & 0 & 2.7 & 0 & 1.54 & 0 & 0.437 \\
\hline halon1202 & 2260 & -13500.0 & 1450 & -8690.0 & 835 & -5060.0 & 391 & -2430.0 & 214 & -1380.0 & 48.8 & -382.0 \\
\hline halon1211 & 6460 & -21700.0 & 6160 & -20700.0 & 5260 & -17800.0 & 3270 & -11200.0 & 1870 & -6600.0 & 425 & -1800.0 \\
\hline halon1301 & 8450 & -10100.0 & 8990 & -10700.0 & 9330 & -11200.0 & 8890 & -10700.0 & 7270 & -8920.0 & 2200 & -3050.0 \\
\hline halon2402 & 4720 & -18900.0 & 4770 & -19200.0 & 4500 & -18200.0 & 3390 & -13800.0 & 2160 & -9040.0 & 506 & -2490.0 \\
\hline hcfc $141 b$ & 4500 & 3800 & 3950 & 3340 & 2960 & 2520 & 1560 & 1350 & 857 & 767 & 195 & 212 \\
\hline hcfc $142 b$ & 7090 & 6870 & 6860 & 6660 & 6010 & 5860 & 3890 & 3840 & 2260 & 2300 & 516 & 627 \\
\hline hcfc22 & 8460 & 8200 & 7730 & 7500 & 6160 & 5940 & 3460 & 3300 & 1920 & 1810 & 438 & 348 \\
\hline hfc125 & 7620 & 7630 & 7750 & 7770 & 7390 & 7370 & 5690 & 5660 & 3690 & 3690 & 872 & 875 \\
\hline hfc134a & 5640 & 5650 & 5290 & 5300 & 4380 & 4420 & 2600 & 2660 & 1460 & 1540 & 333 & 423 \\
\hline hfc143a & 7860 & 7870 & 8250 & 8270 & 8350 & 8320 & 7420 & 7400 & 5570 & 5560 & 1470 & 1470 \\
\hline hfc $152 \mathrm{a}$ & 1850 & 1860 & 1100 & 1110 & 629 & 639 & 294 & 307 & 161 & 174 & 36.7 & 49.4 \\
\hline hfc227ea & 6170 & 6180 & 6360 & 6380 & 6210 & 6250 & 5050 & 5130 & 3440 & 3580 & 835 & 1010 \\
\hline hfc 23 & 11100 & 11100 & 12000 & 12000 & 13100 & 13000 & 14200 & 14000 & 13900 & 13900 & 7900 & 8000 \\
\hline hfc236fa & 6790 & 6800 & 7380 & 7400 & 8010 & 7980 & 8630 & 8610 & 8420 & 8430 & 4610 & 4680 \\
\hline hfc245fa & 5610 & 5620 & 4740 & 4760 & 3400 & 3430 & 1720 & 1770 & 942 & 1000 & 214 & 278 \\
\hline hfc32 & 5760 & 5770 & 4460 & 4480 & 2900 & 2930 & 1390 & 1430 & 760 & 815 & 173 & 227 \\
\hline $\mathrm{hfc} 365 \mathrm{mfc}$ & 4820 & 4830 & 4180 & 4190 & 3090 & 3120 & 1600 & 1650 & 881 & 937 & 200 & 259 \\
\hline hfc 4310 mee & 5290 & 5300 & 5090 & 5110 & 4400 & 4440 & 2790 & 2860 & 1610 & 1690 & 367 & 463 \\
\hline methane & 95.0 & 135 & 85.3 & 122 & 66.1 & 95.3 & 35.8 & 52.9 & 19.7 & 30.2 & 4.44 & 8.33 \\
\hline methyl_bromide & 26.7 & -13200.0 & 15.4 & -7630.0 & 8.75 & -4380.0 & 4.1 & -2100.0 & 2.24 & -1200.0 & 0.511 & -333.0 \\
\hline methyl_chloride & 56.3 & -595.0 & 32.6 & -345.0 & 18.5 & -198.0 & 8.67 & -95.2 & 4.75 & -54.2 & 1.08 & -15.4 \\
\hline nf3 & 12200 & 12200 & 13400 & 13300 & 14700 & 14600 & 16500 & 16400 & 17300 & 17300 & 14200 & 14400 \\
\hline nh3 & 0 & 0 & 0 & 0 & 0 & 0 & 0 & 0 & 0 & 0 & 0 & 0 \\
\hline nitrous_oxide & 286 & 286 & 307 & 308 & 326 & 328 & 330 & 335 & 294 & 302 & 110 & 125 \\
\hline nmvoc & 0 & 36.5 & 0 & 21.2 & 0 & 12.2 & 0 & 5.86 & 0 & 3.33 & 0 & 0.947 \\
\hline nox & 0 & 138 & 0 & 80.1 & 0 & 46.0 & 0 & 22.1 & 0 & 12.6 & 0 & 3.57 \\
\hline nox_avi & 0 & 1620 & 0 & 940 & 0 & 539 & 0 & 259 & 0 & 147 & 0 & 41.9 \\
\hline oc & -1320.0 & -2790.0 & -764.0 & -1620.0 & -435.0 & -930.0 & -204.0 & -446.0 & -112.0 & -254.0 & -25.4 & -72.1 \\
\hline sf6 & 17000 & 17000 & 18700 & 18600 & 20700 & 20600 & 23800 & 23700 & 25700 & 25800 & 27400 & 27800 \\
\hline so2 & -342.0 & -1090.0 & -198.0 & -634.0 & -113.0 & -364.0 & -52.8 & -175.0 & -28.9 & -99.4 & -6.58 & -28.2 \\
\hline so $2 \mathrm{f} 2$ & 7910 & 7920 & 8150 & 8170 & 7950 & 7930 & 6470 & 6440 & 4410 & 4390 & 1070 & 1020 \\
\hline
\end{tabular}



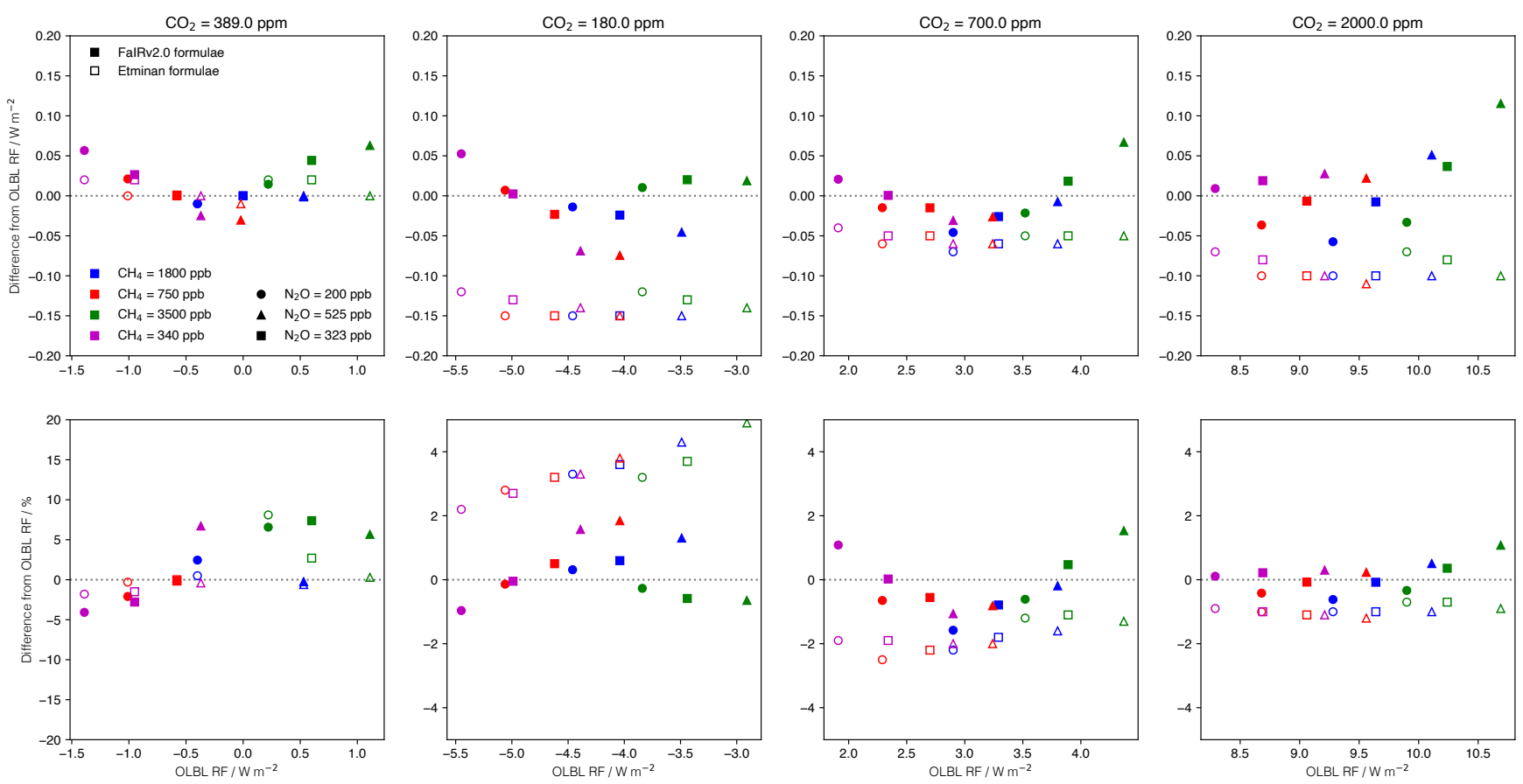

Figure S1. Comparison of the $\mathrm{CO}_{2}, \mathrm{CH}_{4}$ and $\mathrm{N}_{2} \mathrm{O}$ ERF relationships used in FaIRv2.0 to the simple formulae and OLBL data from (Etminan et al., 2016). Top row shows absolute differences, grouped by $\mathrm{CO}_{2}$ concentrations. Bottom row shows differences as a percentage of total forcing, again grouped by $\mathrm{CO}_{2}$ concentration.

\section{Radiative forcing of carbon dioxide, methane and nitrous oxide}

Here we compare the concentration-forcing relationships of $\mathrm{CO}_{2}, \mathrm{CH}_{4}$ and $\mathrm{N}_{2} \mathrm{O}$ used in FaIRv2.0, which exclude the interaction terms between gases, to the standard simple formulae detailed in Etminan et al. (2016). Figure SS1 shows a comparison of the Oslo line-by-line (OLBL) data from Etminan et al. to both the Etminan et al. formulae and those used in FaIRv2.0. The main difference between the relationships used in FaIRv2.0 and those in Etminan et al. is the variance in the error when compared to the OLBL. The FaIRv2.0 relationships have a larger error variance at each $\mathrm{CO}_{2}$ concentration than the Etminan et al. formulae (fig SS1). This is due to the lack of interaction terms, and results in a maximum absolute error of $0.115 \mathrm{~W}$ $\mathrm{m}^{-2}$ at concentrations of $\mathrm{CO}_{2}=2000 \mathrm{ppm}, \mathrm{CH}_{4}=3500 \mathrm{ppm}$, and $\mathrm{N}_{2} \mathrm{O}=525 \mathrm{ppm}$ (the green triangle in the far right-hand side subplot in figure SS1). We believe that in the context of other uncertainties associated with such a high concentration scenario this error is defensible. We note (as was done in Etminan et al.) that the absolute uncertainty in the OLBL calculation is estimated to be $10 \%$ for $\mathrm{CO}_{2}$ and $\mathrm{N}_{2} \mathrm{O}$ and $14 \%$ for $\mathrm{CH}_{4}$. 


\section{CMIP6 data pre-processing}

30 Here we detail the steps taken to remove model drift from and pre-process the CMIP6 data used throughout the study.

\subsection{Drift correction and baseline estimation}

For each model ensemble member, we find the associated parent experiment (a piControl run, not necessarily of the same ensemble member) and branch time in the Dataset Metadata. For each of the abrupt- $4 \mathrm{xCO} 2$ and 1pctCO2 experiments, the model drift is estimated as the slope of a linear regression of the parent experiment against time, starting at the branch year, for a period equal to the duration of the experiment; and the baseline temperature of the experiment is estimated as the value of the regression at the branch point. If the parent experiment ends "before" the experiment ends, then the end of the linear regression is set as the end of the parent experiment and the start moves before the branch point accordingly such that the regression period is still equal to the duration of the experiment. For example, if the experiment is 150 years long, and was branched in year 200 out of a 300-year piControl, the drift would be estimated as the slope of a linear regression over years

40 150-300 of the piControl; and the experiment baseline temperature is the value of the regression line at year 200. This drift correction procedure is applied to both the surface temperature and top-of-atmosphere radiative flux variables for the abrupt$4 \mathrm{xCO} 2$ experiment. For the 1 pctCO2 experiment, we remove the estimated model drift as described above, but estimate the baseline temperature as the intercept of a linear regresion over the first 25 years of the experiment following Sanderson (2020). For the SSP experiment data shown (eg. in figure 9), we do not apply any drift correction.

\section{$45 \quad 3.2$ Other pre-processing}

After applying the drift correction to each ensemble member (if applicable), we then average over all available ensemble members of each model to obtain the data used in fitting parameters, or shown in the figures. The number of ensemble members per model is shown in the table below.

Table S4. Number of ensemble members used per CMIP6 model. 


\begin{tabular}{|c|c|c|c|c|c|c|c|}
\hline model & abrupt- $4 \mathrm{xCO} 2$ & $1 \mathrm{pctCO} 2$ & $\operatorname{ssp} 119$ & $\operatorname{ssp} 126$ & $\operatorname{ssp} 245$ & $\operatorname{ssp} 370$ & $\operatorname{ssp} 585$ \\
\hline ACCESS-CM2 & 1 & 1 & 0 & 3 & 3 & 3 & 3 \\
\hline ACCESS-ESM1-5 & 1 & 1 & 0 & 3 & 3 & 3 & 3 \\
\hline AWI-CM-1-1-MR & 1 & 1 & 0 & 0 & 0 & 0 & 0 \\
\hline BCC-CSM2-MR & 1 & 1 & 0 & 1 & 1 & 1 & 1 \\
\hline BCC-ESM1 & 1 & 1 & 0 & 0 & 0 & 3 & 0 \\
\hline CAMS-CSM1-0 & 2 & 2 & 2 & 2 & 2 & 2 & 2 \\
\hline CESM2 & 1 & 1 & 0 & 3 & 3 & 3 & 3 \\
\hline CESM2-FV2 & 1 & 1 & 0 & 0 & 0 & 0 & 0 \\
\hline CESM2-WACCM & 1 & 1 & 0 & 1 & 3 & 3 & 3 \\
\hline CESM2-WACCM-FV2 & 1 & 1 & 0 & 0 & 0 & 0 & 0 \\
\hline CIESM & 1 & 1 & 0 & 1 & 1 & 0 & 1 \\
\hline CNRM-CM6-1 & 1 & 1 & 0 & 6 & 10 & 6 & 6 \\
\hline CNRM-CM6-1-HR & 1 & 1 & 0 & 1 & 1 & 1 & 1 \\
\hline CNRM-ESM2-1 & 3 & 10 & 5 & 5 & 5 & 5 & 5 \\
\hline CanESM5 & 2 & 6 & 50 & 50 & 50 & 50 & 50 \\
\hline E3SM-1-0 & 1 & 1 & 0 & 0 & 0 & 0 & 0 \\
\hline EC-Earth3-Veg & 1 & 1 & 3 & 5 & 6 & 4 & 5 \\
\hline GFDL-CM4 & 1 & 1 & 0 & 0 & 1 & 0 & 1 \\
\hline GFDL-ESM4 & 1 & 1 & 1 & 1 & 3 & 1 & 1 \\
\hline GISS-E2-1-G & 4 & 5 & 2 & 1 & 10 & 10 & 1 \\
\hline GISS-E2-1-H & 1 & 1 & 0 & 0 & 0 & 0 & 0 \\
\hline GISS-E2-2-G & 1 & 1 & 0 & 0 & 0 & 0 & 0 \\
\hline HadGEM3-GC31-LL & 1 & 4 & 0 & 1 & 4 & 0 & 4 \\
\hline HadGEM3-GC31-MM & 1 & 1 & 0 & 1 & 0 & 0 & 3 \\
\hline INM-CM4-8 & 1 & 1 & 0 & 1 & 1 & 1 & 1 \\
\hline INM-CM5-0 & 1 & 1 & 0 & 1 & 1 & 5 & 1 \\
\hline IPSL-CM6A-LR & 1 & 1 & 6 & 6 & 11 & 11 & 6 \\
\hline KACE-1-0-G & 1 & 1 & 0 & 3 & 3 & 3 & 3 \\
\hline MIROC-ES2L & 1 & 1 & 3 & 3 & 1 & 1 & 1 \\
\hline MIROC6 & 1 & 1 & 1 & 3 & 50 & 3 & 50 \\
\hline MPI-ESM1-2-HR & 1 & 1 & 0 & 2 & 2 & 10 & 2 \\
\hline MPI-ESM1-2-LR & 1 & 1 & 0 & 10 & 10 & 10 & 10 \\
\hline MRI-ESM2-0 & 4 & 2 & 1 & 1 & 5 & 5 & 1 \\
\hline NESM3 & 1 & 1 & 0 & 2 & 2 & 0 & 2 \\
\hline NorCPM1 & 1 & 1 & 0 & 0 & 0 & 0 & 0 \\
\hline NorESM2-LM & 1 & 1 & 0 & 1 & 3 & 3 & 1 \\
\hline NorESM2-MM & 1 & 1 & 0 & 1 & 1 & 1 & 1 \\
\hline SAM0-UNICON & 1 & 1 & 0 & 0 & 0 & 0 & 0 \\
\hline TaiESM1 & 1 & 1 & 0 & 0 & 0 & 0 & 0 \\
\hline UKESM1-0-LL & 1 & 4 & 5 & 13 & 5 & 13 & 5 \\
\hline CAS-ESM2-0 & 0 & 1 & 0 & 0 & 0 & 0 & 0 \\
\hline CMCC-CM2-SR5 & 0 & 1 & 0 & 1 & 1 & 1 & 1 \\
\hline CanESM5-CanOE & 0 & 1 & 0 & 3 & 3 & 3 & 3 \\
\hline FGOALS-f3-L & 0 & 3 & 0 & 1 & 1 & 1 & 1 \\
\hline FGOALS-g3 & 0 & 3 & 0 & 1 & 1 & 1 & 4 \\
\hline FIO-ESM-2-0 & 0 & 3 & 0 & 3 & 3 & 0 & 3 \\
\hline IITM-ESM & 0 & 1 & 0 & 0 & 0 & 0 & 0 \\
\hline MCM-UA-1-0 & 0 & 1 & 0 & 1 & 1 & 1 & 1 \\
\hline MPI-ESM-1-2-HAM & 0 & 1 & 0 & 0 & 0 & 2 & 0 \\
\hline EC-Earth3 & 0 & 0 & 1 & 7 & 16 & 5 & 7 \\
\hline Total models / experiment & 40 & 49 & 12 & 36 & 36 & 33 & 37 \\
\hline
\end{tabular}




\section{Energy balance model parameters}

Using the method described in Cummins et al. (2020), we tune parameters to CMIP6 models for a 3-box version of the energy

55 balance model described in Geoffroy et al. (2013), with the ocean heat uptake efficacy factor as Winton et al. (2010) included.

Table S5. CMIP6 tuned 3-box energy balance model parameters 


\begin{tabular}{|c|c|c|c|c|c|c|c|c|}
\hline & $\gamma$ & $C_{1}$ & $C_{2}$ & $C_{3}$ & $\lambda$ & $\kappa_{2}$ & $\kappa_{3}$ & \\
\hline ACCESS-CM2 & 1.6190 & 3.761 & 11.1100 & $8.269000 \mathrm{e}+01$ & 0.6728 & 3.0130 & 0.6887 & 1.349000 \\
\hline ACCESS-ESM1-5 & 2.7730 & 3.805 & 8.7790 & $8.518000 \mathrm{e}+01$ & 0.6992 & 3.6990 & 0.8399 & 1.565000 \\
\hline AWI-CM-1-1-MR & 4.0820 & 4.225 & 10.6000 & $4.705000 \mathrm{e}+01$ & 1.1690 & 1.9030 & 0.6938 & 1.414000 \\
\hline BCC-CSM2-MR & 2.4290 & 5.157 & 12.2800 & $6.665000 \mathrm{e}+01$ & 0.9237 & 2.3970 & 0.9602 & 1.405000 \\
\hline BCC-ESM1 & 1.9080 & 5.655 & 16.4600 & $7.727000 \mathrm{e}+01$ & 0.8828 & 1.5120 & 0.9629 & 1.283000 \\
\hline CAMS-CSM & 26.8600 & 3.482 & 7.6360 & $5.606000 \mathrm{e}+01$ & 1.8650 & 6.1150 & 0.6972 & 1.241000 \\
\hline CESM2 & 2.6530 & 5.796 & 54.9800 & $9.084000 \mathrm{e}+01$ & 0.4100 & 0.9218 & 0.2440 & 1.358000 \\
\hline CESM2-FV2 & 2.7130 & 3.758 & 7.0540 & $8.885000 \mathrm{e}+01$ & 0.5497 & 3.8790 & 0.9923 & 1.741000 \\
\hline CESM2-WACCM & 2.9960 & 3.733 & 6.1820 & $8.724000 \mathrm{e}+01$ & 0.7171 & 6.8640 & 0.8234 & 1.596000 \\
\hline CESM2-WACCM-FV2 & 2.9130 & 3.371 & 9.4840 & $1.079000 \mathrm{e}+02$ & 0.5598 & 3.5130 & 0.9454 & 1.506000 \\
\hline CIESM & 0.8127 & 5.245 & 11.2100 & $6.379000 \mathrm{e}+01$ & 0.6931 & 2.6240 & 0.9652 & 1.348000 \\
\hline CNRM-CM6-1 & 4.4840 & 4.210 & 16.9000 & $4.185000 \mathrm{e}+02$ & 1.2520 & 1.2360 & 0.4050 & 0.187800 \\
\hline CNRM-CM6 & 6.3770 & 4.159 & 11.4700 & $0 \mathrm{e}+01$ & 1.0180 & 1.9860 & 0.6453 & 0.704900 \\
\hline CNRM-ESN & 3.7910 & 4.709 & 11.0100 & $0 \mathrm{e}+02$ & 0.6646 & 1.5080 & 0.7768 & 700 \\
\hline CanESM5 & 3.6560 & 3.935 & 11.3500 & $0 e+01$ & 0.6359 & 2.1240 & 0.6239 & 1.0 \\
\hline E3SM-1-0 & 3.0600 & 3.654 & 9.5270 & $4.343000 \mathrm{e}+01$ & 0.5771 & 2.3750 & 0.3653 & 5000 \\
\hline EC-Eartl & 26.1800 & 3.413 & 10.0500 & $e+01$ & 0.8413 & 2.4430 & 0.6428 & 000 \\
\hline GFDL-CM4 & 2.7680 & 5.459 & 0.1613 & $7.902000 \mathrm{e}+01$ & 1.0150 & 2.0460 & 1.8810 & 30000 \\
\hline GFDL-ESM4 & 4.0760 & 4.278 & 10.4000 & $1.249000 \mathrm{e}+02$ & 1.5790 & 1.7640 & 0.7689 & 2900 \\
\hline GISS-E2-1-G & 1.0610 & 3.935 & 10.5500 & $1.739000 \mathrm{e}+02$ & 1.5130 & 2.0890 & 1.1660 & 1.068000 \\
\hline GISS & 2.4700 & 4.660 & 24.5300 & $0 e+07$ & 1.6780 & 1.2500 & 85 & 733 \\
\hline GISS & 2.3470 & 3.613 & 10.9600 & $e+02$ & 2.0730 & 1.9590 & 0.5 & 9400 \\
\hline HadGEM3-GC & 3.1200 & 3.817 & 9.3530 & $6.763000 \mathrm{e}+01$ & 0.6122 & 2.7760 & 0.6352 & 1.172000 \\
\hline $\mathrm{HadG}$ & 3.1180 & 3.476 & 14.2200 & $e+01$ & 0.6601 & 2.0260 & 0.7008 & 1.024000 \\
\hline INM-CM4-8 & 2.4800 & 4.306 & 9.5960 & $2.027000 \mathrm{e}+01$ & 1.5800 & 1.8760 & 0.5367 & 1.477000 \\
\hline INM-C & 1.9150 & 4.438 & 11.0000 & $0 \mathrm{e}+01$ & 1.5710 & 1.8660 & 0.5575 & 1.413000 \\
\hline IPSL-CM6A- & 3.2530 & 2.965 & 14.1400 & $6.979000 \mathrm{e}+01$ & 0.6874 & 1.8100 & 0.4502 & 1.374000 \\
\hline KACE-1-0-G & 1.9020 & 0.982 & 11.1700 & $1.180000 \mathrm{e}+02$ & 0.7261 & 27.7700 & 0.9366 & 1.246000 \\
\hline MIROC6 & 2.2020 & 3.781 & 17.0300 & $1.779000 \mathrm{e}+02$ & 1.4470 & 1.7410 & 1.1070 & 1.176000 \\
\hline MPI-ESM1 & 2.9040 & 5.201 & 23.2600 & $7.145000 \mathrm{e}+01$ & 1.1860 & 1.5940 & 1.0890 & 1.531000 \\
\hline MPI-ESM1-2-LR & 2.2430 & 6.466 & 49.4100 & $1.575000 \mathrm{e}+06$ & 1.7270 & 0.8626 & 0.2811 & 0.407300 \\
\hline MRI-ESM2-0 & 1.6290 & 4.907 & 10.8100 & $9.678000 \mathrm{e}+01$ & 1.1190 & 2.3480 & 1.3580 & 1.279000 \\
\hline NESM3 & 2.8310 & 2.516 & 19.2000 & $1.146000 \mathrm{e}+02$ & 0.9230 & 1.0370 & 0.4592 & 0.757500 \\
\hline NorCPM1 & 1.1690 & 7.160 & 35.9900 & $5.470000 \mathrm{e}+07$ & 1.5480 & 1.2060 & 0.4017 & 0.507700 \\
\hline SAM0-UNICON & 2.4450 & 4.578 & 6.3010 & $1.111000 \mathrm{e}+02$ & 1.0320 & 2.6630 & 1.0110 & 1.274000 \\
\hline TaiESM1 & 2.0330 & 5.074 & 8.9460 & $8.662000 \mathrm{e}+01$ & 0.8827 & 2.2900 & 0.9088 & 1.229000 \\
\hline UKESM1-0-LL & 3.5760 & 2.984 & 11.2400 & $6.968000 \mathrm{e}+01$ & 0.6533 & 2.6840 & 0.6245 & 1.138000 \\
\hline MIROC-ES2L & 0.0140 & 10.040 & 21.1900 & $2.550000 \mathrm{e}+02$ & 1.6510 & 0.9538 & 0.8993 & 0.594300 \\
\hline NorESM2-LM & 1.0280 & 3.730 & 175.5000 & $1.448000 \mathrm{e}+00$ & 0.7494 & 3.2310 & 1.3360 & 445.100000 \\
\hline NorESM2-MM & 1.3830 & 4.129 & 128.3000 & $5.881000 \mathrm{e}-01$ & 1.9660 & 1.4410 & 0.7010 & 207.500000 \\
\hline
\end{tabular}



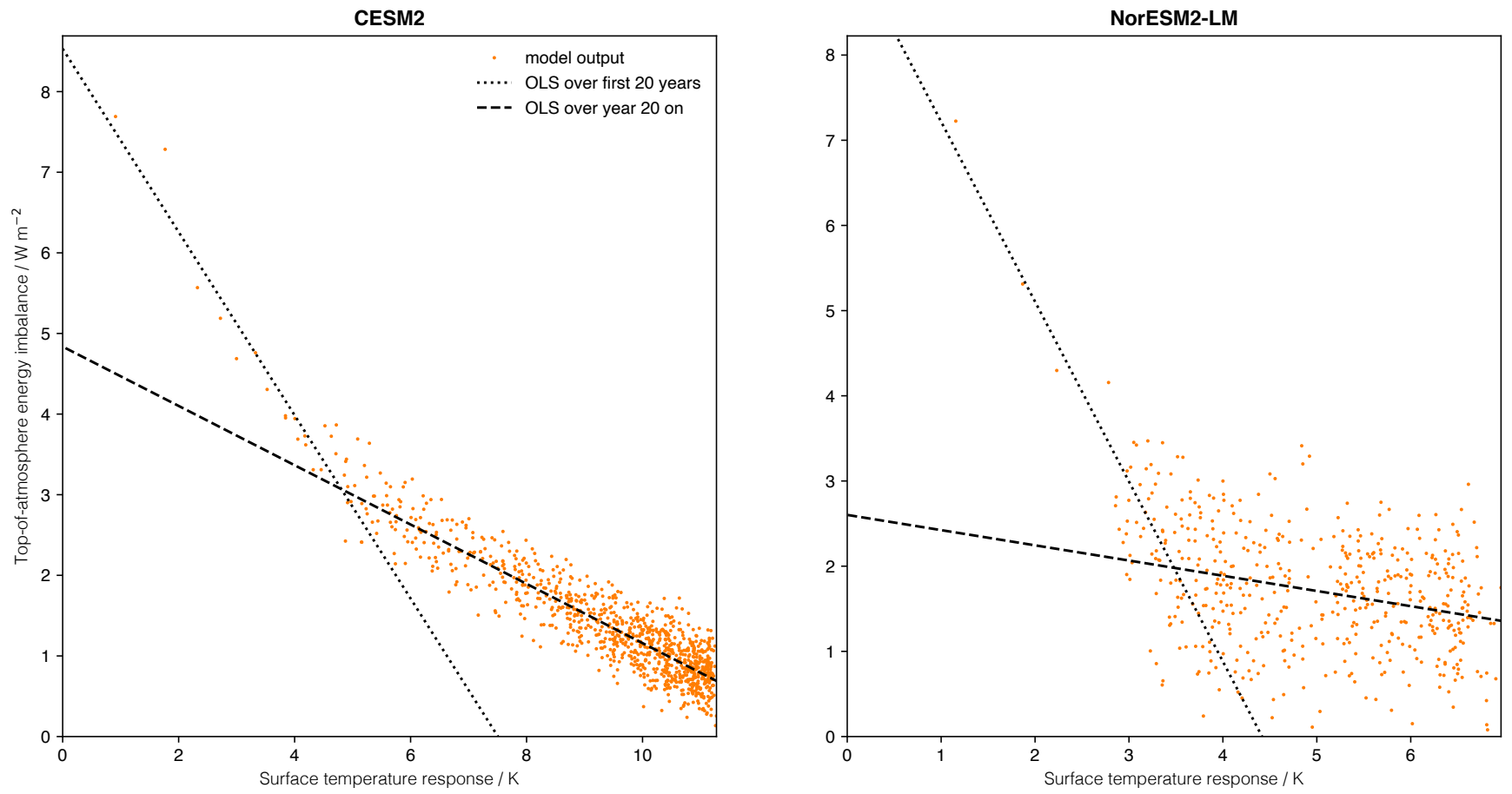

Figure S2. Gregory plots over the abrupt-4xCO2 experiment for CESM2 and NorESM2-LM. The dotted line shows the result of a linear regression over the first 20 years of the experiment, while the dashed line shows the result if instead the regression is carried out from year 20 onwards.

\section{Gregory plots for CESM2 and NorESM2-LM}

In fig SS2 we show Gregory plots (Gregory et al., 2002) for CESM2 and NorESm2-LM to support the discussion in section $60 \quad 3.1$. 


\section{Global Warming Index calculation}

The Global Warming Index follows the methodology in Haustein et al. (2016), but updates several components. We use the mean of 5 datasets for the observed warming, with associated uncertainty taken from the HadCRUT4 ensemble (Lenssen et al., 2019; Cowtan and Way, 2014; Vose et al., 2012; Morice et al., 2011; Rohde et al., 2013). We then generate 4000 realisations of historical ERF as follows. For all forcings excluding aerosol forcing, we take the best-estimate historical timeseries from Smith (2020), and scale them by factors drawn from the distributions detailed in table 6 . For aerosol forcing, we take the bestestimate historical timeseries of ERFaci and ERFari, and scale them by scaling factors drawn from a skew-normal distribution that matches the quantiles of the constrained ERFaci and ERFari distributions stated in table 4 of Smith et al. (2020). We consider 18 different response model parameterisations, spanning the ranges of possible realised warming fraction (Millar et al., 2015) and response timescale (Geoffroy et al., 2013). Finally, we include uncertainty due to internal variability through timeseries from the piControl experiment of different CMIP6 models, rejecting models with a too large drift; resulting in 51 different representations of internal variability. Combining these sources of uncertainty results in a 367,200,000 member ensemble of the global warming index, from which we derive the 5-95\% ranges used to constrain the FULL ensemble in the main text.

$75 \quad 7$ ALTERNATIVE ensemble variable correlations 

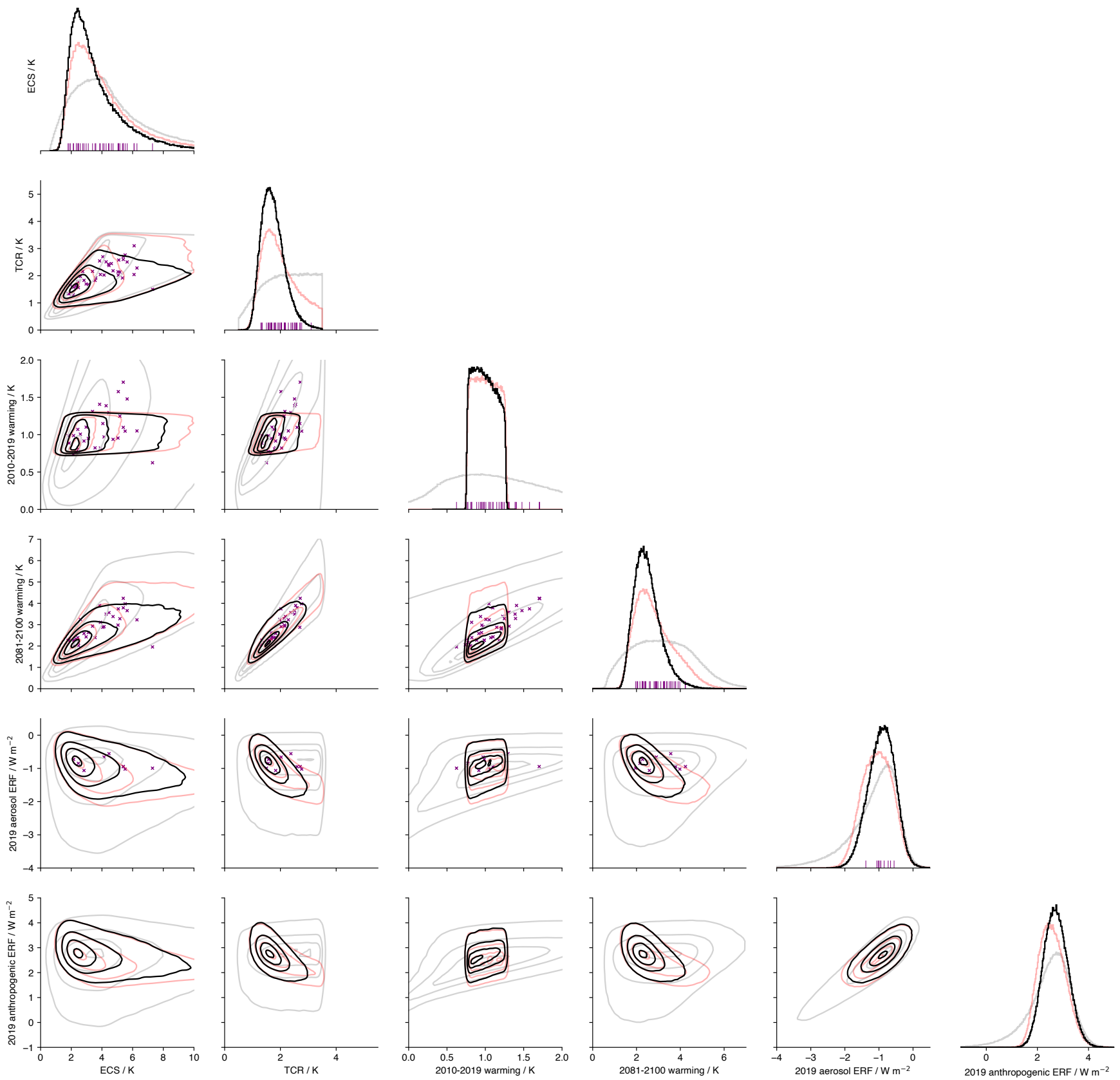

Figure S3. Corner plot depiction of the ALTERNATIVE prior and posterior ensembles as described in the main text. Diagonal plots show marginal probability density functions of each key variable; the prior shown in grey, ALTERNATIVE in black, and the prior constrained by the current level of warming only in red. Subdiagonal plots show contour plots of gaussian kernel density estimates of joint probability density. Contours show indicate regions containing 95, 67, 33 and $5 \%$ of the ensemble members. Purple crosses and lines indicate the positions of individual CMIP6 models. 
Code and data availability. The code used to produce the figures is publicly available at https://github.com/njleach/GIR. However, we stress that the code here is not a model release. This update to the FaIR model will be made available at https://github.com/OMS-NetZero/FAIR when fully integrated and tested. All data used in this study is publicly available at the relevant cited sources. 


\section{References}

Cowtan, K. and Way, R. G.: Coverage bias in the HadCRUT4 temperature series and its impact on recent temperature trends, Quarterly Journal of the Royal Meteorological Society, 140, 1935-1944, https://doi.org/10.1002/qj.2297, http://doi.wiley.com/10.1002/qj.2297, 2014.

Cummins, D. P., Stephenson, D. B., and Stott, P. A.: Optimal Estimation of Stochastic Energy Balance Model Parameters, Journal of Climate, pp. JCLI-D-19-0589.1, https://doi.org/10.1175/JCLI-D-19-0589.1, http://journals.ametsoc.org/doi/10.1175/JCLI-D-19-0589.1, 2020.

Etminan, M., Myhre, G., Highwood, E. J., and Shine, K. P.: Radiative forcing of carbon dioxide, methane, and nitrous oxide: A significant revision of the methane radiative forcing, Geophysical Research Letters, 43, 12,614-12,623, https://doi.org/10.1002/2016GL071930, http: //doi.wiley.com/10.1002/2016GL071930, 2016.

Geoffroy, O., Saint-Martin, D., Olivié, D. J. L., Voldoire, A., Bellon, G., Tytéca, S., Geoffroy, O., Saint-Martin, D., Olivié, D. J. L., Voldoire, A., Bellon, G., and Tytéca, S.: Transient Climate Response in a Two-Layer Energy-Balance Model. Part I: Analytical Solution and Parameter Calibration Using CMIP5 AOGCM Experiments, Journal of Climate, 26, 1841-1857, https://doi.org/10.1175/JCLI-D-1200195.1, http://journals.ametsoc.org/doi/abs/10.1175/JCLI-D-12-00195.1http://journals.ametsoc.org/jcli/article-pdf/26/6/1841/4002942/ jcli-d-12-00195\{_\}1.pdf, 2013.

Gregory, J. M., Stouffer, R. J., Raper, S. C. B., Stott, P. A., and Rayner, N. A.: An Observationally Based Estimate of the Climate Sensitivity, Journal of Climate, 15, 3117-3121, https://doi.org/10.1175/1520-0442(2002)015<3117:AOBEOT>2.0.CO;2, http://journals.ametsoc.org/ doi/abs/10.1175/1520-0442\{\%\}282002\{\%\}29015\{\%\}3C3117\{\%\}3AAOBEOT\{\%\}3E2.0.CO\{\%\}3B2, 2002.

Haustein, K., L Otto, F. E., Uhe, P., Schaller, N., Allen, M. R., Hermanson, L., Christidis, N., McLean, P., and Cullen, H.: Real-time extreme weather event attribution with forecast seasonal SSTs, Environ. Res. Lett, 11, 64 006, https://doi.org/10.1088/1748-9326/11/6/064006, 2016.

Lenssen, N. J. L., Schmidt, G. A., Hansen, J. E., Menne, M. J., Persin, A., Ruedy, R., and Zyss, D.: Improvements in the uncertainty model in the Goddard Institute for Space Studies Surface Temperature (GISTEMP) analysis, Journal of Geophysical Research: Atmospheres, p. 2018JD029522, https://doi.org/10.1029/2018JD029522, https://onlinelibrary.wiley.com/doi/abs/10.1029/2018JD029522, 2019.

Meinshausen, M., Vogel, E., Nauels, A., Lorbacher, K., Meinshausen, N., Etheridge, D. M., Fraser, P. J., Montzka, S. A., Rayner, P. J., Trudinger, C. M., Krummel, P. B., Beyerle, U., Canadell, J. G., Daniel, J. S., Enting, I. G., Law, R. M., Lunder, C. R., O\&apos;Doherty, S., Prinn, R. G., Reimann, S., Rubino, M., Velders, G. J. M., Vollmer, M. K., Wang, R. H. J., and Weiss, R.: Historical greenhouse gas concentrations for climate modelling (CMIP6), Geoscientific Model Development, 10, 2057-2116, https://doi.org/10.5194/gmd-10-20572017, https://www.geosci-model-dev.net/10/2057/2017/, 2017.

Millar, R. J., Otto, A., Forster, P. M., Lowe, J. A., Ingram, W. J., and Allen, M. R.: Model structure in observational constraints on transient climate response, Climatic Change, 131, 199-211, https://doi.org/10.1007/s10584-015-1384-4, http://link.springer.com/10.1007/ s10584-015-1384-4, 2015.

Morice, C. P., Kennedy, J. J., Rayner, N. A., Jones, P. D., P., M. C., J., K. J., A., R. N., and D., J. P.: Quantifying uncertainties in global and regional temperature change using an ensemble of observational estimates: The HadCRUT4 data set, Journal of Geophysical Research: Atmospheres, 117, https://doi.org/10.1029/2011JD017187, https://agupubs.onlinelibrary.wiley.com/doi/abs/10.1029/2011JD017187https: //www.metoffice.gov.uk/hadobs/hadcrut4/HadCRUT4\{_\}accepted.pdf, 2011.

Rohde, R., Muller, R. A., Jacobsen, R., Muller, E., Perlmutter, S., Rosenfeld, A., Wurtele, J., Groom, D., and Wickham, C.: A New Estimate of the Average Earth Surface Land Temperature Spanning 1753 to 2011, Geoinformatics \& Geostatistics: An Overview, 1, 1, https://doi.org/10.4172/2327-4581.1000101, http://dx.doi.org/10.4172/2327-4581.1000101, 2013. 
Sanderson, B.: Relating climate sensitivity indices to projection uncertainty, Earth System Dynamics, 11, 721-735, https://doi.org/10.5194/esd-11-721-2020, https://esd.copernicus.org/articles/11/721/2020/, 2020.

Smith, C.: Effective Radiative Forcing Time Series from the Shared Socioeconomic Pathways, https://doi.org/10.5281/ZENODO.3973015, https://zenodo.org/record/3973015, 2020.

Smith, C. J., Harris, G., Palmer, M. D., Bellouin, N., Myhre, G., Schulz, M., Golaz, J.-C., Ringer, M., Storelvmo, T., and Forster, P. M.: Energy Budget Constraints on the Time History of Aerosol Forcing and Climate Sensitivity, Journal of Geophysical Research: Atmospheres, in submiss, 2020.

Vose, R. S., Arndt, D., Banzon, V. F., Easterling, D. R., Gleason, B., Huang, B., Kearns, E., Lawrimore, J. H., Menne, M. J., Peterson, T. C., Reynolds, R. W., Smith, T. M., Williams, C. N., Wuertz, D. B., Vose, R. S., Arndt, D., Banzon, V. F., Easterling, D. R., Gleason, B., Huang, B., Kearns, E., Lawrimore, J. H., Menne, M. J., Peterson, T. C., Reynolds, R. W., Smith, T. M., Jr., C. N. W., and Wuertz, D. B.: NOAA's Merged Land-Ocean Surface Temperature Analysis, Bulletin of the American Meteorological Society, 93, 1677-1685, https://doi.org/10.1175/BAMS-D-11-00241.1, http://journals.ametsoc.org/doi/abs/10.1175/BAMS-D-11-00241.1, 2012.

Winton, M., Takahashi, K., and Held, I. M.: Importance of Ocean Heat Uptake Efficacy to Transient Climate Change, Journal of Climate, 23, 2333-2344, https://doi.org/10.1175/2009JCLI3139.1, http://journals.ametsoc.org/jcli/article-pdf/23/9/2333/3968777/2009jcli3139\{_\}1. pdf, 2010. 